drainage systems may not be feasible for some sink designs and could be costly and labor intensive. Thus, we are currently investigating several simpler approaches to achieve the same effect.

Acknowledgments. We would like to thank the staff of the Cleveland VA Medical Center's Engineering Service for assistance in designing and installing sink valves.

Financial support. This work was supported by the Department of Veterans Affairs.

Conflicts of interest. C.J.D. has received research funding from Clorox, GOJO, Pfizer, Avery Dennison, PDI, and Boehringer Laboratories. All other authors report no potential conflicts.

\section{References}

1. Kizny Gordon AE, Mathers AJ, Cheong EYL, et al. The hospital water environment as a reservoir for carbapenem-resistant organisms causing hospital-acquired infections-a systematic review of the literature. Clin Infect Dis 2017 May 15;64:1435-1444.
2. Jencson AL, Cadnum JL, Piedrahita C, Donskey CJ. Hospital sinks are a potential nosocomial source of Candida infections. Clin Infect Dis 2017;65:1954-1955.

3. Livingston SH, Cadnum JL, Gestrich S, Jencson AL, Donskey CJ. A novel sink drain cover prevents dispersal of microorganisms from contaminated sink drains. Infect Control Hosp Epidemiol 2018;39: 1254-1256.

4. Parkes LO, Hota SS. Sink-related outbreaks and mitigation strategies in healthcare facilities. Curr Infect Dis Rep 2018;20:42.

5. Kotay S, Chai W, Guilford W, Barry K, Mathers AJ. Spread from the sink to the patient: in situ study using green fluorescent protein (GFP)expressing Escherichia coli to model bacterial dispersion from hand washing sink trap reservoirs. Appl Environ Microbiol 2017;83(8):pii: e03327-16.

6. Klick JM, du Moulin GC, Hedley-Whyte J, Teres D, Bushnell LS, Feingold DS. Prevention of gram-negative bacillary pneumonia using polymyxin aerosol as prophylaxis. II. Effect on the incidence of pneumonia in seriously ill patients. J Clin Invest 1975;55:514-519.

7. Gbaguidi-Haore H, Varin A, Cholley P, Thouverez M, Hocquet D, Bertrand X. A bundle of measures to control an outbreak of Pseudomonas aeruginosa associated with P-trap contamination. Infect Control Hosp Epidemiol 2018;39:164-169.

\title{
A novel color additive for bleach wipes indicates surface coverage and contact time to improve thoroughness of cleaning
}

\author{
Kevin Tyan BA, Katherine Jin BA and Jason Kang BSc \\ Kinnos, Brooklyn, New York
}

To the Editor-Healthcare-associated infections (HAIs) exact a heavy toll on the US healthcare system, affecting $\sim 1.7$ million patients and resulting in direct costs of up to $\$ 45$ billion each year. ${ }^{1}$ Institutions are heavily emphasizing frequent disinfection of hightouch surfaces to prevent transmission to patients because contaminated surfaces are known to be reservoirs for nosocomial pathogens. ${ }^{2}$ In particular, hospitals have increasingly adopted the approach of daily cleaning with ready-to-use bleach wipes to combat $C$. difficile infections (CDIs). Daily cleaning with a sporicidal agent was demonstrated to be the most effective single intervention against CDI and asymptomatic colonization, ${ }^{3}$ while one hospital reported a reduction of $85 \%$ in $\mathrm{CDI}$ following the implementation of daily bleach wipe cleaning in all patient rooms. ${ }^{4}$

However, effective disinfection requires proper technique, adequate training, and constant monitoring. ${ }^{5}$ Significant human error in disinfectant wiping practices has been well documented, with personnel missing high-touch surfaces, overusing single wipes, and inadvertently transferring pathogens between surfaces, or drying off the applied disinfectant before the necessary wet-contact time. ${ }^{5}$ Periodic assessments of cleaning compliance through adenosine triphosphate (ATP) bioluminescence assays and fluorescent marker systems may help mitigate human error, but improvements cannot be sustained without permanent systematic changes and constant

Author for correspondence: Kevin Tyan, 760 Parkside Avenue, Suite 215, Brooklyn, NY 11226. E-mail: kevin@kinnos.us

Cite this article: Tyan K, et al. (2019). A novel color additive for bleach wipes indicates surface coverage and contact time to improve thoroughness of cleaning. Infection Control \& Hospital Epidemiology 2019, 40, 256-258. doi: 10.1017/ice.2018.323 feedback. This assertion is evidenced by a report in which environmental services (EVS) staff improved their cleaning performance from $52 \%$ to $83 \%$ after implementing fluorescent marker monitoring but regressed toward the baseline (57\%) after monthly feedback ceased. ${ }^{6}$ Current methods for quality control monitoring are retrospective and often are only intermittently performed; thus, it may be challenging to translate to timely feedback for EVS staff. To sustain a high level of cleaning compliance, a unique approach is needed, one that provides direct and immediate feedback to workers.

A novel attachment to bleach wipe containers, the Highlight Wipes Lid (Kinnos, Brooklyn, NY) administers a blue indicator onto dispensed bleach wipes to provide real-time visual feedback of the thoroughness of surface coverage and the passage of contact time. ${ }^{7}$ As shown in Fig. 1A, the device consists of (1) a reusable lid that attaches onto standard containers of commercially available bleach wipes, and (2) a disposable cartridge containing the Highlight blue liquid additive and pre-installed batteries. A user presses the button to dispense individual wipes imbued with the blue indicator through the front face of the battery-powered lid. This dispensing mechanism was designed for ease of use and prevention of bleach splash-back that typically occurs when manually pulling wipes through standard lid orifices. Furthermore, the lid automatically retracts hanging wipes back into the canister after a period of inactivity to prevent drying of the bleach wipe, loss of efficacy, and wastage of the productproblems common in currently used bleach wipes.

Figure $1 \mathrm{~B}$ compares the visibility of surface coverage using bleach wipes alone and bleach wipes dispensed through the Highlight Wipes Lid. When wiped on a standard bedside rail 
A

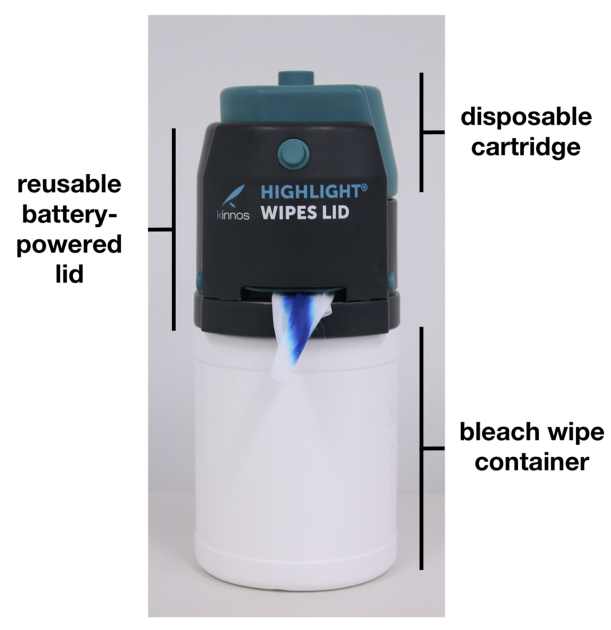

B

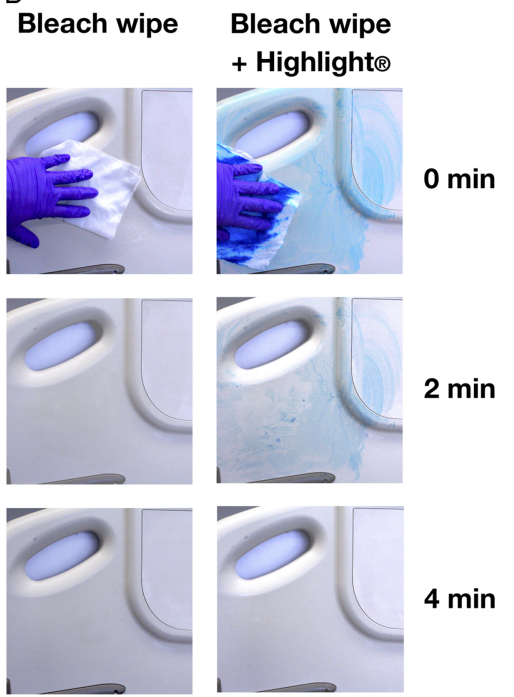

Fig. 1. Overview of the Highlight Wipes Lid system. (A) The reusable battery-powered lid attaches directly on top of standard containers of commercially available bleach wipes. A disposable cartridge inserts on top of the reusable lid and contains the Highlight additive, which is administered onto each bleach wipe as it is dispensed. (B) Comparison of visibility of surface coverage 0,2 , and 4 minutes after wiping a bedside rail using bleach wipes alone versus bleach wipes with Highlight.

(Hill-Rom, Chicago, IL), the surface coverage of bleach wipes alone was difficult to see across all time points. In contrast, bleach wipes with Highlight provided a bright blue color that was still visible 2 minutes after wiping but completely faded to clear after 4 minutes. By leaving a blue trace where bleach has been wiped onto a surface, Highlight provides users with a means to monitor their own cleaning technique at the point of use. The fading away of blue indicator after $\sim 4$ minutes allows users to monitor the passage of wet-contact time required for bleach wipes. The transient blue color can effectively deter personnel from prematurely wiping away the bleach, as has been previously reported, ${ }^{5}$ and it prevents patients and other staff from interacting with recently wiped surfaces that have not reached the necessary contact time.

To determine whether Highlight Wipes Lids improve the thoroughness of bleach wipe cleaning over current methods, a prospective study was conducted in a 500-bed academic medical center across 11 medical and intensive care unit (ICU) inpatient wards. Routine terminal cleaning was performed by EVS staff using ready-to-use bleach wipes (Sani-Cloth bleach germicidal disposable wipes, PDI Healthcare, Orangeburg, NY). The staff was then provided with Highlight Wipes Lids to implement in their terminal cleaning. The study was performed over a 55-week period from February 2017 to February 2018. A designated infection control staff member randomly sampled high-touch surfaces following terminal cleaning using an ATP bioluminescence assay system (SystemSURE Plus ATP Cleaning Verification System; Hygiena, Camarillo, CA). A total of 1,020 high-touch surfaces were sampled, including bedside rails, toilet flush handles, room sinks, bathroom door handles, and television remote controls. Based on manufacturer recommendations and institutional guidelines, the relative light unit (RLU) benchmarks used to assess thoroughness of cleaning were pass ( $\leq 50 \mathrm{RLU})$ and fail (>50 RLU) ${ }^{8}$ Cleaning with bleach wipes alone yielded a failure rate of $5.70 \%$ (54 of 947 samples) with an average RLU value (mean \pm SD) of $18 \pm 137$, whereas the introduction of Highlight with bleach wipes resulted in no failures (0 of 73 samples) with an average RLU value of $14 \pm 10$. Although the average RLU values for both wiping strategies fell below the cleanliness threshold of 50 RLU, the high standard deviation for bleach wipes alone indicates that a significant number of high-touch surfaces were entirely missed from cleaning, resulting in outlier values due to high bioburden. In comparison, every high-touch surface sampled in rooms cleaned with Highlight passed below the cleanliness threshold of 50 RLU.

Our results suggest that the implementation of a real-time visual feedback system for disinfectant wipes can improve both the thoroughness of surface coverage and cleaning compliance. In fact, a previous study found that the use of Highlight indicator in spray-based bleach disinfectants helped healthcare workers correctly identify surfaces where bleach had been applied. ${ }^{9}$ We have previously demonstrated that Highlight not only can be added into bleach disinfectants without compromising antimicrobial efficacy and skin safety ${ }^{10}$ but also acts to reduce bleach corrosion on materials like stainless steel. ${ }^{7}$

Our study has some limitations. Visibility of the bleach wipe coverage was only compared on a light surface (bedside rail), although a recent study assessed the blue Highlight indicator on a variety of healthcare surfaces (including black countertops) and found enhanced visibility in most cases. ${ }^{9}$ In addition, the sample size for bleach wipes with Highlight was small due to the limited scope of the device pilot study in the hospital. Nonetheless, the perfect pass rate of the high-touch samples cleaned with Highlight as well as the lower and more consistent RLU values suggest that these results are compelling. Additional assessments of this novel cleaning strategy should focus on other metrics including fluorescent marker removal, usability in the hospital setting, healthcare worker feedback, and patient outcomes.

Acknowledgments. We thank Cathy Korn, Mohamed Dassouli, Deborah Gregson, David Maffeo, and all other staff at Boston Medical Center who helped us conduct the hospital study.

Financial support. Kinnos, Inc., provided the product for testing but did not participate in the design or execution of the hospital study.

Conflicts of interest. K.T., K.J., and J.K. are founders and shareholders of Kinnos, Inc., and have patents pending on the Highlight technology. 


\section{References}

1. Dick AW, Perencevich EN, Pogorzelska-Maziarz M, et al. A decade of investment in infection prevention: a cost-effectiveness analysis. Am J Infect Control 2015;43:4-9.

2. Weber DJ, Anderson D, Rutala WA. The role of the surface environment in healthcare-associated infections. Curr Opin Infect Dis 2013;26:338-344.

3. Barker AK, Alagoz O, Safdar N. Interventions to reduce the incidence of hospital-onset Clostridium difficile infection: an agent-based modeling approach to evaluate clinical effectiveness in adult acute care hospitals. Clin Infect Dis 2018;66:1192-1203.

4. Orenstein R, Aronhalt KC, McManus JE, et al. A targeted strategy to wipe out Clostridium difficile. Infect Control Hosp Epidemiol 2011;32:1137-1139.

5. Cadnum JL, Hurless KN, Kundrapu S, Donskey CJ. Transfer of Clostridium difficile spores by non-sporicidal wipes and improperly used hypochlorite wipes: practice + product $=$ perfection. Infect Control Hosp Epidemiol 2013;34:441-442.
6. Fitzgerald T, Sholtz LA, Marion N, Turner P, Carling PC, Rupp ME. Maintenance of environmental services cleaning and disinfection in the ICU after a performance improvement project. Am J Infect Control 2012;40:e159.

7. Tyan K, Jin K, Kang J. Novel colour additive for bleach disinfectant wipes reduces corrosive damage on stainless steel. J Hosp Infect (in press).

8. Lower and upper RLU limits for ATP monitoring programs. Hygiena website. https://www.hygiena.com/hc-getstarted.html. Published 2016. Accessed November 21, 2018.

9. Mustapha A, Cadnum JL, Alhmidi H, Donskey CJ. Evaluation of novel chemical additive that colorizes chlorine-based disinfectants to improve visualization of surface coverage. Am J Infect Control 2018;46:119-121.

10. Tyan K, Kang J, Jin K, Kyle AM. Evaluation of the antimicrobial efficacy and skin safety of a novel color additive in combination with chlorine disinfectants. Am J Infect Control 2018;46:1254-1261.

\title{
A regional collaboration between competing healthcare systems to establish influenza season parameters
}

\author{
David H. Priest MD, MPH ${ }^{1}$, Susan L. DeCamp-Freeze RN, MBA ${ }^{1}$, Cynthia B. Snider MD, MPH ${ }^{2}$, Melissa G. Morgan \\ MSN, RN ${ }^{2}$, Misty D. Garner MLS ${ }^{3}$, Laurence B. Givner MD ${ }^{4}$, Catherine L. Passaretti $\mathrm{MD}^{5}$ and \\ Andrea B. McQuaigue $\mathrm{RN}^{6}$ \\ ${ }^{1}$ Department of Infection Prevention, Novant Health, Winston-Salem, North Carolina, ${ }^{2}$ Department of Infection Prevention, Cone Health, Greensboro, North \\ Carolina, ${ }^{3}$ Department of Infection Prevention, Randolph Health, Asheboro, North Carolina, ${ }^{4}$ Department of Pediatrics, Wake Forest Baptist Health, Winston- \\ Salem, North Carolina, ${ }^{5}$ Department of Infection Prevention, Atrium Health, Charlotte, North Carolina and ${ }^{6}$ Department of Infection Prevention, High Point \\ Regional UNC Health Care, High Point, North Carolina
}

To the Editor-Collaborative public health partnerships between competing healthcare system hospitals are uncommon in today's healthcare environment, despite their potential for improving community health. ${ }^{1-4}$ One potential area of collaboration between hospital systems is management of influenza season parameters and messaging team members and the community. Traditionally, acute-care facilities make independent decisions regarding influenza season parameters. In geographic areas with multiple healthcare systems, the lack of coordination in influenza-season decision making can lead to a local patchwork of policies and messages. These different public health messages can be confusing to patients and the public, potentially affecting patient care, visitor access, and patient satisfaction.

We established a regional collaboration between multiple healthcare systems that emphasized information sharing and unified messaging to the public and local media. A weekly conference call was initiated that included 6 competing healthcare entities located in the piedmont region of North Carolina: Novant Health, Cone Health, Randolph Health, Wake Forest Baptist Health, Atrium Health, and High Point Regional UNC Health Care. In total, these systems represent 36 acute-care facilities covering $\sim 9,000$ square miles with $\sim 4$ million people. ${ }^{5}$ All of these systems have mandatory influenza vaccination programs for team

Author for correspondence: David H. Priest, MD, MPH, 1381 Westgate Center Drive, Winston-Salem, NC 27103. E-mail: dhpriest@novanthealth.org

Cite this article: Priest DH, et al. (2019). A regional collaboration between competing healthcare systems to establish influenza season parameters. Infection Control \& Hospital Epidemiology 2019, 40, 258-260. doi: 10.1017/ice.2018.297 members, and 4 of the systems have mandatory masking programs for team members unable to be vaccinated (ie, Novant Health, Atrium Health, High Point Regional UNC Health Care, and Randolph Health). The call included infection prevention representatives, marketing/communication professionals, nursing leaders, physicians, and hospital administrators. Each healthcare system reported their local emergency department influenza-likeillness (ILI) rate for comparison with the other systems. Facility differences were noted based on the geographic location of the hospitals. Emergency department (ED) ILI data were obtained using the North Carolina Disease Event Tracking and Epidemiologic Collection Tool (NC DETECT), a statewide syndromic surveillance system. ${ }^{6}$ Collective decisions were made to establish parameters for influenza season, which were then communicated through internal and external communication pathways.

Influenza season was declared when the ILI rate in North Carolina hospital ED visits reached $\geq 5 \%$ and ended when the ILI rate declined back to $<5 \%$ (Fig. 1), considering the general ILI trend. For the 2017-2018 influenza season, the start date was December 27, 2017, and the end date was March 30, 2018. Declaring when influenza season started and ended was more important for those healthcare systems that require mandatory masking of team members who do not receive an influenza vaccination, as this was the date in which mandatory masking began. In the past, for some healthcare systems, the influenza season started and ended using predetermined dates such as October 1 (start) and March 31 (end). Although the time between these predetermined dates typically encompassed the influenza season, 